Canadian

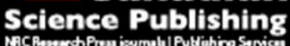

Applied Physiology, Nutrition, and Metabolism Physiologie appliquée, nutrition et métabolisme

\title{
Sprint interval and moderate-intensity cycling training differentially affect adiposity and aerobic capacity in overweight young-adult women.
}

\begin{tabular}{|r|l|}
\hline Journal: & Applied Physiology, Nutrition, and Metabolism \\
\hline Manuscript ID & apnm-2016-0240.R2 \\
\hline Manuscript Type: & Article \\
\hline Date Submitted by the Author: & 25-Jul-2016 \\
\hline Complete List of Authors: & $\begin{array}{l}\text { Higgins, Simon; University of Georgia, Kinesiology } \\
\text { Fedewa, Michael; University of Georgia, Department of Kinesiology; } \\
\text { University of Alabama, Department of Kinesiology } \\
\text { Hathaway, Elizabeth; University of Georgia, Department of Kinesiology } \\
\text { Schmidt, Michael; University of Georgia, Department of Kinesiology } \\
\text { Evans, Ellen; University of Georgia, }\end{array}$ \\
\hline Keyword: & $\begin{array}{l}\text { High-intensity, Moderate-intensity, Central Adiposity, Equal Energy } \\
\text { Expenditure, body composition < body composition }\end{array}$ \\
\hline \multicolumn{2}{|c}{} \\
\hline
\end{tabular}




\title{
Sprint interval and moderate-intensity cycling training differentially affect adiposity and aerobic capacity in overweight young-adult women.
}

\author{
Authors: Simon Higgins ${ }^{1}$; Michael V. Fedewa ${ }^{1,2}$; Elizabeth D. Hathaway ${ }^{1}$; Michael D. \\ Schmidt $^{1}$; Ellen M. Evans ${ }^{1}$
}

Corresponding Author: Simon Higgins, Department of Kinesiology, Office 101D, Ramsey Center, 330 River Road, University of Georgia, Athens, GA 30602-6554. Telephone: 706-4616776. E-mail: sh@uga.edu

\footnotetext{
Author Affiliations:

${ }^{1}$ Department of Kinesiology, University of Georgia, 330 River Road, Athens, GA 30602-6554.

Email: Simon Higgins - sh@uga.edu, Elizabeth D. Hathaway - lizhath@uga.edu, Michael D.

Schmidt-schmidtm@uga.edu, and Ellen M. Evans-emevans@uga.edu.

${ }^{2}$ Department of Kinesiology, The University of Alabama, 2003 Moore Hall, Box 870312,

Tuscaloosa, AL 35487-0231. E-mail: Michael V. Fedewa - mvfedewa@ua.edu
} 


\begin{abstract}
Purpose: To examine the effects of sprint interval training (SIT) and moderate-intensity continuous cycle training (MICT), with equal estimated energy expenditure during training, on body composition and aerobic capacity. Methods: Body composition measured via dual-energy $\mathrm{x}$-ray absorptiometry and aerobic capacity $\left(\mathrm{VO}_{2 \text { peak }}\right)$ were assessed following 6 weeks of training in previously inactive overweight/obese young women $\left(\mathrm{n}=52,20.4 \pm 1.5 \mathrm{yr}, 30.3 \pm 4.5 \mathrm{~kg} \cdot \mathrm{m}^{-2}\right.$, $67.3 \%$ white). Training was performed in a group-exercise format that mimicked cycling classes offered by commercial fitness facilities, and included 3 weekly sessions of either 30-second 'allout' sprints followed by 4 minutes of active recovery (SIT), or continuous cycling at 60-70\% heart rate reserve to expend a similar amount of energy. Results: Participants were randomized to SIT or MICT, attended a similar number of sessions $(15.0 \pm 1.5$ sessions vs. $15.8 \pm 1.9$ sessions, $\mathrm{P}=.097)$ and expended a similar amount of energy $\left(541.8 \pm 104.6 \mathrm{~kJ} \cdot \mathrm{session}^{-1} \mathrm{vs} .553 .5 \pm 138.1\right.$ $\mathrm{kJ} \cdot$ session $\left.^{-1}, \mathrm{P}=.250\right)$. Without significant changes in body mass $(\mathrm{P}>.05)$, greater relative reductions occurred in SIT than in MICT in total fat mass $(3.6 \pm 5.6 \%$ vs. $0.6 \pm 3.9 \%, \mathrm{P}=.007)$ and android fat mass $(6.6 \pm 6.9 \%$ vs. $0.7 \pm 6.5 \%, \mathrm{P}=.002)$. Aerobic capacity $\left(\mathrm{mL} \cdot \mathrm{kg}^{-1} \cdot \mathrm{min}^{-1}\right)$ increased significantly following both interventions $(\mathrm{P}<.05)$, but the relative increase was 2 -fold greater in SIT than in MICT $(14.09 \pm 10.31 \%$ vs. $7.06 \pm 7.81 \%, \mathrm{P}<.001)$. Conclusion: Sprint interval cycling reduces adiposity and increases aerobic capacity more than continuous moderate-intensity cycling of equal estimated energy expenditure in overweight/obese young women.
\end{abstract}

Keywords: High-intensity, Moderate-intensity, Central Adiposity, Equal Energy Expenditure, Body Composition. 


\section{Introduction}

High-intensity interval training (HIIT) is a mode of exercise that is characterized by brief, intermittent bursts of vigorous activity, interspersed by periods of rest or low-intensity exercise (Gibala et al., 2012). HIIT has been investigated in many populations with consistent improvements in body composition (Boutcher, 2011; Heydari et al., 2012) and aerobic capacity (Gist et al., 2013; Sloth et al., 2013; Trilk et al., 2011; Weston et al., 2014). It has been suggested that HIIT may be more efficient than traditional moderate-intensity continuous exercise training protocols (MICT), producing greater adaptation from a similar training duration, comparable to that produced by high-volume endurance-type training (Gillen and Gibala, 2014). The popularity of HIIT reaches beyond the physiological adaptations (Gibala and McGee, 2008), with research suggesting that it may also be more enjoyable than MICT (Bartlett et al., 2011). These advantages give HIIT the potential to have a greater adherence rate than conventional exercise interventions (Jung et al., 2015).

A category of HIIT which employs bouts of efforts at an even higher intensity and power output than traditional HIIT protocols is sprint interval training (SIT). A typical SIT protocol includes 30-second 'all out' efforts followed by 4 minutes of active recovery at a low-intensity (Freese et al., 2015; Gibala et al., 2006). The effects of SIT have been explored in healthy populations (Hazell et al., 2014; Macpherson et al., 2011; Whyte et al., 2010), and in some clinical populations (Gibala et al., 2012). With regard to overweight/obese women who are underrepresented in the SIT literature, positive effects on aerobic capacity and cardiovascular function, post-exercise blood lipids, and perceived health status and mood have been reported (Freese et al., 2014; Freese et al., 2015; Trilk et al., 2011). While studies have shown that the benefits of SIT are similar to those of continuous exercise involving a greater volume of work in 
different populations (Gist et al., 2013; Kessler et al., 2012), the feasibility of overweight/obese women adhering to these 'all out' efforts is unclear, as are the relative effects of the two types of training when energy expenditure during training is equal. It is likely that in most studies, greater effects of the higher exercise intensity and power output of SIT have been offset by greater energy expenditure in the continuous training exercise regimens to which they have been compared, resulting in relatively similar changes in outcomes of interest.

The ability of continuous exercise training to facilitate weight loss and positive body composition change, in the absence of dietary compensation, is well established (Swift et al., 2014). Studies examining SIT suggest similar effects (Gibala and McGee, 2008), with the potential for greater benefits to body composition (Boutcher, 2011) than MICT. The few reported SIT interventions in women have used training durations of 6-15 weeks (Hazell et al., 2014; Trapp et al., 2008) and reported significant reductions in weight and adiposity. Moreover, there is a small amount of research comparing SIT to MICT in inactive overweight/obese women; one study to do so did not ensure equal exercise energy expenditure during training (Keating et al., 2014). Indeed, total energy expenditure during an exercise session is a key mechanism responsible for weight and body composition changes (Swift et al., 2014); however, few studies involving SIT have controlled for this factor making it difficult to compare the independent effects of exercise intensity on body composition. One study to investigate the effects of exercise intensity on body composition showed that high-intensity continuous exercise, matched on energy expenditure with a lower-intensity regimen, led to greater losses of visceral fat and abdominal subcutaneous fat over 16 weeks in obese middle-aged women (Irving et al., 2008); however, both regimens used continuous exercise protocols. Contrary to these findings, some have argued that it is the volume, not the intensity, of exercise that leads to reductions in 
abdominal adiposity (Ross et al., 2015), thus, the potential for exercise intensity to mediate magnitude of fat loss warrants further research.

It is also well established that aerobic exercise training improves aerobic capacity (Garber et al., 2011; Gormley et al., 2008). Recent work comparing the relative effects of SIT and MICT interventions on aerobic capacity in sedentary adults over 6 (Burgomaster et al., 2008; Cocks et al., 2013), 12 (Keating et al., 2014), and 15 weeks (Trapp et al., 2008) reported similar significant improvements in aerobic capacity regardless of training mode. Interestingly, although SIT interventions lasted up to 4 months, SIT interventions of just 2 weeks have also significantly improved aerobic capacity by an average of 9.5\% (Whyte et al., 2010). The positive relationship between the intensity of aerobic work and the magnitude of improvement in aerobic capacity is accepted (Gormley et al., 2008); however, if the greater increase in aerobic capacity following higher intensity work holds true with a SIT protocol of an even higher intensity and intermittent nature is unknown, especially when work or exercise energy expenditure is equal. As interval training becomes increasingly popular, more research is needed regarding the effect of exercise intensity/pattern on body composition and aerobic capacity when using a SIT protocol, controlling for energy expenditure, especially in overweight/obese women as SIT is understudied in this cohort.

Thus in this context, the primary aim of this study was to examine the relative effects of 6 weeks of SIT compared to a MICT protocol of equal estimated energy expenditure on body composition and aerobic capacity in inactive overweight/obese young women. We hypothesized that the magnitude of changes in body composition and aerobic capacity would be greater in SIT compared to MICT under training conditions of equal estimated energy expenditure. 


\section{Methods}

Study Population and Recruitment. This analysis was part of a parent study examining the effect of SIT and MICT protocols on markers of metabolic syndrome risk. Women $(n=60)$ were recruited via email and print advertising and excluded if they were: current smokers (within 6 months), physically active ( $\geq 30$ minutes of physical activity, $\geq 2$ days per week), not aged 18 $24 \mathrm{yr}$, not enrolled at the University, $<25 \mathrm{~kg} \cdot \mathrm{m}^{-2} \mathrm{BMI}$, varsity athletes, currently pregnant, or had been pregnant within the previous 12 months. Individuals with any health condition that could have been exacerbated by the exercise protocols, or for which the exercise may be unsafe were not enrolled. The Institutional Review Board of the university approved all aspects of the protocol and all participants provided written informed consent prior to participation.

Study Design and Exercise Interventions. This research study used a parallel-arm design with participants stratified on BMI and subsequently randomized to a SIT or MICT group. All sessions were performed on a friction-loaded stationary cycle ergometer which has the ability to estimate energy expenditure based on power output (Keiser, Keiser M3 Indoor Cycle, Fresno, California). Heart rate (HR) during exercise, ratings of perceived exertion (RPE), and estimated energy expenditure from the Keiser ergometers were recorded during each exercise session to ensure compliance with the training protocol. Participants assigned to the SIT group began each exercise session with a 4-minute warm-up at a low resistance and pedal rate, followed by repeated 30-second 'all out' sprints interspersed with 4 minutes of active recovery pedaling against minimal resistance at a low pedal frequency. This exercise stimulus equated to 2.5-3.5 minutes of near-maximal effort interspersed with 16-28 minutes of recovery, along with a brief cool-down period following each session. Training progression increased the number of sprint repetitions from 5 during weeks 1 and 2, to 6 sprints during weeks 3 and 4, and 7 sprints during 
weeks 5 and 6. Participants assigned to the MICT group were instructed to cycle continuously at an intensity of $60-70 \%$ heart rate reserve (HRR) for 20-30 minutes, with the duration of training sessions systematically increased to maintain equal estimated energy expenditure between groups. Training protocols were performed 3 times weekly in a supervised group-training format which were led by trained research staff and mimicked cycling classes offered by commercial fitness facilities.

Weight Status and Body Composition. Standing height was measured by a stadiometer (Seca 242, SECA Corp, Hamburg, Germany) to the nearest $0.1 \mathrm{~cm}$. Body mass was measured with a digital scale (Tanita WB-110A class III, Tanita Corporation, Tokyo, Japan) to the nearest $0.1 \mathrm{~kg}$. Subsequently, BMI was calculated as mass divided by height squared $\left(\mathrm{kg} \cdot \mathrm{m}^{-2}\right)$. Whole body and regional composition including absolute and relative adiposity (\%Fat) and mineral free soft tissue mass, designated herein as fat free mass (FFM), were measured via dual x-ray absorptiometry (DXA; Lunar iDXA, v 11.30.062, GE Healthcare, Madison, Wisconsin).

Aerobic Capacity. Aerobic capacity $\left(\mathrm{VO}_{2 \text { peak }}\right)$ was measured with a maximal exercise test whereby participants pedaled continuously on an electronically braked cycle ergometer, with the workload increasing every 3 minutes in a graded manner (Lode Excalibur Sport 2000, Lode B.V., Groningen, Netherlands). Oxygen uptake was measured using a computerized indirect calorimetry system (ParvoMedics True Max 2400; ParvoMedics, Sandy, UT). HR was measured continuously throughout the test (Polar FT1; Polar Electro, Kempele, Finland). The test was terminated when traditional termination criteria were met for respiratory exchange ratio, HR, RPE, volitional fatigue, or pedal rate dropped below $50 \mathrm{rpm}$. Aerobic capacity is expressed in absolute terms $\left(\mathrm{L} \cdot \mathrm{min}^{-1}\right)$, relative to body mass $\left(\mathrm{mL} \cdot \mathrm{kg}^{-1} \cdot \mathrm{min}^{-1}\right)$, and relative to fat free mass $\left(\mathrm{mL} \cdot \mathrm{kgFFM}^{-1} \cdot \mathrm{min}^{-1}\right)$. 


\section{Habitual Energy Expenditure and Reported Energy Intake. Energy expenditure was}

measured objectively over a minimum 3-day continuous period using the Actiheart monitor (CamNtech, USA), which uses both HR and an omnidirectional accelerometer movement sensor. Reported energy intake was measured using the Automated Self-Administered 24-hour Recall (ASA24), a Web-based tool modeled on the USDA's Automated Multiple Pass Method (Subar et al., 2012). Energy intake and expenditure data were collected over 3-day periods including 2 weekdays and 1 weekend day at baseline and during weeks 5-6 of the intervention (endpoint), and are reported as average values.

Statistical Analysis. Statistical analyses were performed using SPSS for Windows (SPSS 22.0, Chicago, IL). After testing for violation of assumptions using standard procedures, baseline differences between groups were assessed using independent t-tests. Pre- to post-intervention changes in outcome variables were analysed using a 2x2 (GROUP*TIME) repeated measures analysis of covariance (ANCOVA) with GROUP included as the between training group factor, and TIME included as the within factor. Baseline values for outcome variables served as a covariate. Post hoc pairwise comparisons with a Bonferroni adjustment for repeated contrasts were used to identify specific treatment effects. As this is a secondary analysis from a parent study, an a priori power analysis was not possible. However, given the sample size $(\mathrm{n}=52)$ in the designated primary (fat mass) and secondary $\left(\mathrm{VO}_{2 \text { peak }}\left[\mathrm{mL} \cdot \mathrm{kg}^{-1} \cdot \mathrm{min}^{-1}\right]\right)$ outcomes of interest, post hoc power anaylsis revealed statistical power well in excess of 0.90 for both outcomes. To further characterize the magnitude of change in SIT relative to the comparison group (MICT), effect sizes (ES) are reported as unbiased standardized mean change using the equation $E S_{s m}=$ $\left(\bar{X}_{G 1}-\bar{X}_{G 2}\right) /\left(s_{\text {control }}\right)$ (Lipsey and Wilson, 2001) which was subsequently corrected for sample size bias using hedges unbiased effect size estimate $E S^{\prime}{ }_{s m}=\left[1-\frac{3}{4 N-9}\right] E S_{s m}$ (Hedges, 
1981). Statistical significance was set at an $\alpha$ level of $\mathrm{P}<.05$. All data are expressed as mean \pm standard deviation $(\mathrm{M} \pm \mathrm{SD})$, unless otherwise indicated.

\section{Results}

Participant Characteristics and Adherence. Of the participants that completed the intervention $(n=60)$, seven participants were excluded based on $<70 \%$ attendance $(n=5$ SIT; $n=2$ MICT) and one was excluded from analysis as an outlier (baseline fat mass values $>3$ SD from the mean). Thus, the cohort $(n=52)$ of young women $(20.4 \pm 1.5 \mathrm{yr})$ were on average obese $(30.3$ $\left.\pm 4.5 \mathrm{~kg} \cdot \mathrm{m}^{-2}\right)$ and mostly white $(67.3 \%)$ paralleling university racial/ethnic demographics $(19.2 \%$ Black, 5.8\% Asian, 1.9\% Hispanic, and 5.8\% other). Groups did not differ on baseline characteristics $(\mathrm{P}>.05$; data not shown). Session attendance (15.0 \pm 1.5 sessions vs. $15.8 \pm 1.9$ sessions, $\mathrm{P}=.097)$ and average energy expenditure $\left(541.8 \pm 104.6 \mathrm{~kJ} \cdot\right.$ session $^{-1}$ vs. $553.5 \pm 138.1$ $\mathrm{kJ} \cdot \mathrm{session}^{-1}, \mathrm{P}=.250$ ) was not different between SIT and MICT. No adverse events were reported within either intervention group. Available complete total daily energy expenditure data $(n=44)$ indicated no change from pre- to post- intervention in either SIT $\left(10,859.6 \pm 1,406.7 \mathrm{~kJ} \cdot \mathrm{d}^{-1} \mathrm{vs}\right.$. $\left.10,719.4 \pm 1,170.7 \mathrm{~kJ} \cdot \mathrm{d}^{-1}, \mathrm{P}=.544\right)$ or MICT $\left(10,506.9 \pm 1,315.0 \mathrm{~kJ} \cdot \mathrm{d}^{-1}\right.$ vs. $10,591.8 \pm 1,195.4$ $\left.\mathrm{kJ} \cdot \mathrm{d}^{-1}, \mathrm{P}=.956\right)$, with no significant GROUP*TIME interaction $(\mathrm{P}=.632)$. Complete dietary data $(\mathrm{n}=51)$ indicated a main effect of time $(\mathrm{P}<.001)$ but no GROUP*TIME interaction $(\mathrm{P}=.308)$. Post hoc analysis revealed no change in response to SIT $\left(7,930.4 \pm 2,203.7 \mathrm{~kJ} \cdot \mathrm{d}^{-1}\right.$ vs. $7,688.5 \pm$ $\left.2,165.6 \mathrm{~kJ} \cdot \mathrm{d}^{-1}, \mathrm{P}=.356\right)$, but a significant reduction in $\operatorname{MICT}\left(8,367.2 \pm 2,869.4 \mathrm{~kJ} \cdot \mathrm{d}^{-1} \mathrm{vs} .7,388.5\right.$ $\left.\pm 2,051.0 \mathrm{~kJ} \cdot \mathrm{d}^{-1}, \mathrm{P}=.013\right)$.

Mass \& Body Composition. Changes in outcome variables are expressed in relative terms within the main text and in absolute terms within Table 1. No changes in body mass occurred in response to either of the exercise interventions $(\mathrm{ES}=.07, \mathrm{P}=.118)$. A significant GROUP*TIME 
interaction was found for reductions in total fat mass $(\mathrm{ES}=.12, \mathrm{P}=.007)$, with SIT reducing total fat mass by $3.6 \%(\mathrm{P}<.001)$ compared to $0.6 \%(\mathrm{P}=.757)$ in MICT (Table 1, Figure $1 \mathrm{~A})$. A significant interaction was also found for changes in $\%$ Fat $(\mathrm{ES}=.14, \mathrm{P}=.013)$, with further analysis indicating a $1 \%$ decrease in $\operatorname{SIT}(\mathrm{P}<.001)$ and no change $(-0.3 \%, \mathrm{P}=.315)$ in MICT. Similarly, a significant GROUP*TIME interaction $(\mathrm{ES}=.16, \mathrm{P}=.002)$ existed indicating that exercise intensity had differential effects on android fat mass with a $6.6 \%$ reduction in SIT and no change $(-0.7 \%)$ in MICT. Total body FFM did not change differentially $(E S=.00, P=.983)$ or within either group (both $\mathrm{P}>.05)$. The change in leg FFM was similar in both groups $(\mathrm{ES}=.03$, $\mathrm{P}=.557)$, increasing by $2.0 \%$ in $\operatorname{MICT}(\mathrm{P}=.001)$ and $1.9 \%$ in $\operatorname{SIT}(\mathrm{P}<.001)$.

Aerobic Capacity. A significant GROUP*TIME interaction $(\mathrm{ES}=.48, \mathrm{P}<.001)$ existed with SIT demonstrating a 2-fold $(14.1 \%)$ increase in $\mathrm{VO}_{2 \text { peak }}\left(\mathrm{mL} \cdot \mathrm{kg}^{-1} \cdot \mathrm{min}^{-1}\right)$ compared to the 7.1\% increase in MICT (Table 1, Figure 1B). Similar interactions and changes were observed for $\mathrm{VO}_{2 \text { peak }}$ expressed in absolute terms $\left(\mathrm{L} \cdot \mathrm{min}^{-1} ; \mathrm{ES}=.49, \mathrm{P}=.001\right)$ and relative to $\mathrm{FFM}\left(\mathrm{mL} \cdot \mathrm{kgFFM}^{-}\right.$ $\left.{ }^{1} \cdot \min ^{-1} ; \mathrm{ES}=.46, \mathrm{P}=.002\right)$.

\section{Discussion}

The effect of exercise intensity on body composition and aerobic capacity in overweight/obese young women was examined with 6-week SIT and MICT interventions involving equal estimated exercise energy expenditure. In agreement with our hypothesis, the major findings were: 1) exercise training intensity/pattern differentially affected adiposity, with SIT eliciting significantly greater losses of total and central fat mass compared to MICT, and 2) although both interventions led to a significant increase in aerobic capacity, SIT led to a nearly 2-fold greater increase in $\mathrm{VO}_{2 \text { peak }}$ than in MICT, regardless of expression. 
Body Mass and Composition. The absence of substantial change in body mass is not surprising in light of the low energy expenditure of each session $\sim 550 \mathrm{~kJ}$, totaling $\sim 10,000 \mathrm{~kJ}$ over the 6-week intervention. However, the reported reductions in total and central fat mass in favor of SIT are mirrored by an absolute reduction in body mass which neared significance $(p=.06)$ and was not seen following MICT. In support of these findings, a recent study using a 6week running SIT protocol of a similar duration and intensity to the present study (Hazell et al., 2014), reported a $1.2 \mathrm{~kg}$ reduction in fat mass with only a $0.5 \mathrm{~kg}$ reduction in body mass, despite presumably low exercise energy expenditure and no changes to dietary energy intake. The significant reduction in energy intake within MICT of $\sim 1000 \mathrm{~kJ} /$ day in the absence of body composition change, along with the $\sim 2500 \mathrm{~kJ}$ discrepancy between energy intake and expenditure reported by participants is paradoxical and highlights the limitations of methodologies for measuring free living energy intake. Indeed, part of the variance in body composition change could be accounted for by changes in energy balance not captured by these methodologies; however, these limitations are present in all such studies, similarly affecting intervention groups. Moreover, most studies are consistent in their findings of reduced adiposity following similar SIT protocols (Hazell et al., 2014; Macpherson et al., 2011), along with other forms of high intensity interval work (Gillen et al., 2013; Heydari et al., 2012), which speaks to the validity of the findings and strength of the lipolytic effect of high intensity interval training. However, one study comparing SIT and MICT failed to show a beneficial effect of SIT, nor one which was greater than MICT (Keating et al., 2014). In overweight/obese adults, 12 weeks of MICT reduced android fat by $2.7 \%$, whereas SIT led to no reductions in adiposity $(\mathrm{P}=.04)$ (Keating et al., 2014). Notably, this intervention showed similar discrepancies in energy intake and expenditure to the current data, and did not have equal work or exercise energy expenditure 
between groups which could have played a role in the contradictory findings, as by the final week of training, exercise duration was 2-fold greater in MICT compared to SIT (Keating et al., 2014).

Though reductions in adiposity have been consistently noted following SIT (Hazell et al., 2014; Macpherson et al., 2011), much debate exists regarding the mechanisms that drive the lipolytic effect of SIT (Boutcher, 2011), and how they might differ from MICT. One theory is that an intensity driven hormone release occurs with SIT, which augments lipolysis (Bracken et al., 2009; Nevill et al., 1996; Williams et al., 2013; Zouhal et al., 2008). Nevill et al., showed that elevations in growth hormone were linked to peak power output, with greater concentrations occurring following SIT than endurance work (Nevill et al., 1996). Furthermore, increased production of the catecholamines epinephrine and norepinephrine in comparison to MICT has also been reported in some studies (Bracken et al., 2009; Williams et al., 2013; Zouhal et al., 2008), but not others (Sevits et al., 2013). Indeed, these hormones are important contributors to lipolysis, however, in the case of increased catecholamine production, the high rate of lipolysis does not appear to translate into augmented fat oxidation, leading only to high re-esterification rates (Mora-Rodriguez and Coyle, 2000; Williams et al., 2013). More research is needed to define the relationships among training regimen type, hormones released at supramaximal intensities, and subsequent fat oxidation.

A second theory that has been considered to explain favorable changes in adiposity is a higher excess post-exercise oxygen consumption (EPOC) throughout the remainder of the day. Although the positive relationship between exercise intensity and EPOC is well accepted (Bahr and Sejersted, 1991), investigations into EPOC following SIT suggest that energy expenditure is similar to that of MICT protocols (Kelly et al., 2013; Sevits et al., 2013; Williams et al., 2013), 
with changes in total daily energy expenditure being attributable to the energy cost of exercise and any differences between protocols being too small in magnitude ( $\sim 6-15 \%$ of the total oxygen cost of exercise) to explain the changes in body composition (Kelly et al., 2013; LaForgia et al., 2006). It is noteworthy that the recovery period between sprints is accompanied by an increased EPOC, which contributes to overall energy expenditure above that which is captured by measurement methods other than calorimetry (LaForgia et al., 2006). As such, the use of Keiser ergometers to estimate energy expenditure and the lack of oxygen uptake data post-training limits the mechanistic interpretation of our data as we cannot confirm differences in EPOC; however notably, these limitations are balanced by the greater translational utility of this exercise intervention.

A third, more recent hypothesis focuses on the potentially satiating effect of SIT, reducing perceptions of hunger (Deighton et al., 2013; Williams et al., 2013), and in some cases energy intake (Alkahtani et al., 2014; Sim et al., 2015). However, there remains question as to whether a change in measured appetite translates to a reduction in energy intake, as malalignment between the two have been reported (Deighton et al., 2013). As we were unable to measure specific aspects of these proposed mechanisms, the explanation of greater fat loss following SIT remains elusive and warrants further investigation.

Aerobic Capacity. Studies using a similar SIT protocol that employed an equal work or exercise energy expenditure design between intervention groups are sparse. One study compared 6 sessions of 4-7 SIT bouts over 2 weeks to a MICT group working at an intensity below their ventilatory threshold, with an equal estimated energy expenditure. This study found no significant improvements in $\mathrm{VO}_{2 \text { peak }}$ in MICT but 7.5\% $\left(\mathrm{L} \cdot \mathrm{min}^{-1}\right)$ and $6.7 \%\left(\mathrm{~mL} \cdot \mathrm{kg}^{-1} \cdot \mathrm{min}^{-1}\right)$ increases with SIT (Bailey et al., 2009). Conversely, a study by Keating et al. showed that in 
overweight adults, 12 weeks of training improved aerobic capacity to a similar extent in SIT $(22.3 \pm 3.5 \%)$ and MICT $(23.8 \pm 3.0 \%)$ groups (Keating et al., 2014). A number of potential moderators may explain the conflicting results from previous SIT and MICT comparisons including study duration, MICT intensity, and lack of energy expenditure control in each exercise group subsequently leading to different training volumes and limiting the interpretation of the findings.

A recent meta-analysis summarizing the effect of SIT on aerobic capacity in young healthy participants suggested that a SIT intervention would be expected to induce on average an $8 \%$ increase in $\mathrm{VO}_{2 \max }$ relative to non-exercise control groups (Cohen's $d=.69$ ) and that changes would not be different from a moderate-to-vigorous-intensity continuous training intervention of a similar duration (Cohen's $d=.04$ ) (Gist et al., 2013). With the similarities between Cohen's $d$ and the ES used in this analysis, an effect size of $\mathrm{ES}=.48$ for increases in $\mathrm{VO}_{2 \text { peak }}\left(\mathrm{mL} \cdot \mathrm{kg}^{-1} \cdot \mathrm{min}^{-1}\right)$ suggests that the SIT protocol used in this study had a stronger effect on aerobic capacity than reported in previous literature. However, this and similar reviews did not explore relationships between equal exercise energy expenditure or work-matched interventions and outcomes of interest, which makes the comparison of results difficult (Gist et al., 2013; Weston et al., 2014).

SIT has induced rapid improvements in both aerobic capacity and muscular oxidative potential, with a meta-analysis showing several studies of a 2-week duration significantly improving $\mathrm{VO}_{2 \max }$ (Sloth et al., 2013). Within this review, it was suggested that the time-course of adaptations to SIT may differ from MICT, the former eliciting peripheral adaptations at a much faster rate than the latter. This has implications when looking at study duration and the comparison of SIT and MICT interventions, pointing towards interventions with a shorter duration being more likely to show superior improvements in aerobic capacity with SIT. Further 
research is needed to elucidate the effects of longer duration SIT interventions, with MICT comparison groups, to identify the different time-course of adaptations as current reviews have failed to resolve this issue (Gist et al., 2013; Sloth et al., 2013; Weston et al., 2014).

As suggested, another factor influencing the comparability of SIT and continuous exercise results is the differing intensity of the continuous exercise comparison groups within the current literature, with intensities of $65-80 \% \mathrm{VO}_{2 \max }$ (Esfarjani and Laursen, 2007; Keating et al., 2014; Rowan et al., 2012), along with $\mathrm{HR}_{\max }$ based methodologies (Sandvei et al., 2012). This range of 'moderate-to-vigorous' intensities could logically elicit differential improvements in aerobic capacity as previous research has shown that the intensity of continuous exercise is generally linearly related to improvements in $\mathrm{VO}_{2 \max }$ (Gormley et al., 2008). In the context of this study, it is possible that the MICT exercise intensity was not high enough to elicit the same magnitude of changes in just 6 weeks; however, further research looking at equivalent exercise stimuli is needed to address this issue.

Conclusion. In summary, compared to a MICT protocol in which the same estimated energy was expended, 6 weeks of SIT elicited significantly greater reductions in total and central adiposity, and increases in aerobic capacity in overweight/obese young women. Aerobic capacity improved 2-fold more with SIT compared to MICT, with increases in $\mathrm{VO}_{2 \text { peak }}$ being greater than expected based on recent reviews. Our findings suggest that SIT is an effective means to rapidly improve body composition and aerobic capacity in overweight/obese young women when performed in a supervised group-training format. 
Acknowledgments: No sources of funding were used in preparation of this manuscript. The authors declare no conflict of interest. Data collection was performed by SH, MVF, and EDH. Review of literature and statistical analyses were performed by SH. Manuscript preparation and revision were performed by SH, MVF, EDH, MDS, and EME. Preparation of the final document for submission was performed by SH and EME. 


\section{References:}

Alkahtani, S. A., Byrne, N. M., Hills, A. P., and King, N. A. 2014. Interval training intensity affects energy intake compensation in obese men. Int J Sport Nutr Exerc Metab. 24(6): 595-604. doi:10.1123/ijsnem.2013-0032

Bahr, R., and Sejersted, O. M. 1991. Effect of intensity of exercise on excess postexercise O2 consumption. Metabolism. 40(8): 836-841. doi:10.1016/0026-0495(91)90012-L

Bailey, S. J., Wilkerson, D. P., Dimenna, F. J., and Jones, A. M. 2009. Influence of repeated sprint training on pulmonary $\mathrm{O} 2$ uptake and muscle deoxygenation kinetics in humans. J Appl Physiol. 106(6): 1875-1887. doi:10.1152/japplphysiol.00144.2009

Bartlett, J. D., Close, G. L., MacLaren, D. P., Gregson, W., Drust, B., and Morton, J. P. 2011. Highintensity interval running is perceived to be more enjoyable than moderate-intensity continuous exercise: implications for exercise adherence. J Sports Sci. 29(6): 547-553. doi:10.1080/02640414.2010.545427

Boutcher, S. H. 2011. High-intensity intermittent exercise and fat loss. J Obes. 2011: 868305. doi:10.1155/2011/868305

Bracken, R. M., Linnane, D. M., and Brooks, S. 2009. Plasma catecholamine and nephrine responses to brief intermittent maximal intensity exercise. Amino Acids. 36(2): 209-217. doi:10.1007/s00726008-0049-2

Burgomaster, K. A., Howarth, K. R., Phillips, S. M., Rakobowchuk, M., Macdonald, M. J., McGee, S. L., et al. 2008. Similar metabolic adaptations during exercise after low volume sprint interval and traditional endurance training in humans. J Physiol. 586(1): 151-160. doi:10.1113/jphysiol.2007.142109

Cocks, M., Shaw, C.S., Shepherd, S.O., Fisher, J., Ranasinghe, A.M., Barker, T.A., et al. 2013. Sprint interval and endurance training are equally effective in increasing muscle microvascular density 
and eNOS content in sedentary males. J Physiol. 591(3): 641-656.

doi:10.1113/jphysiol.2012.239566

Deighton, K., Barry, R., Connon, C. E., and Stensel, D. J. 2013. Appetite, gut hormone and energy intake responses to low volume sprint interval and traditional endurance exercise. Eur J Appl Physiol. 113(5): 1147-1156. doi:10.1007/s00421-012-2535-1

Esfarjani, F., and Laursen, P. B. 2007. Manipulating high-intensity interval training: effects on VO2max, the lactate threshold and 3000 m running performance in moderately trained males. J Sci Med Sport. 10(1): 27-35. doi:10.1016/j.jsams.2006.05.014

Freese, E. C., Acitelli, R. M., Gist, N. H., Cureton, K. J., Evans, E. M., and O'Connor, P. J. 2014. Effect of six weeks of sprint interval training on mood and perceived health in women at risk for metabolic syndrome. J Sport Exerc Psychol. 36(6): 610-618. doi:10.1123/jsep.2014-0083

Freese, E. C., Gist, N. H., Acitelli, R. M., McConnell, W. J., Beck, C. D., Hausman, D. B., et al. 2015. Acute and chronic effects of sprint interval exercise on postprandial lipemia in women at-risk for the metabolic syndrome. J Appl Physiol. 118(7): 872-879. doi:10.1152/japplphysiol.00380.2014

Garber, C. E., Blissmer, B., Deschenes, M. R., Franklin, B. A., Lamonte, M. J., Lee, I. M., et al. 2011. American College of Sports Medicine position stand. Quantity and quality of exercise for developing and maintaining cardiorespiratory, musculoskeletal, and neuromotor fitness in apparently healthy adults: guidance for prescribing exercise. Med Sci Sports Exerc. 43(7): 13341359. doi:10.1249/MSS.0b013e318213fefb

Gibala, M. J., Little, J. P., van Essen, M., Wilkin, G. P., Burgomaster, K. A., Safdar, A., et al. 2006. Short-term sprint interval versus traditional endurance training: similar initial adaptations in human skeletal muscle and exercise performance. J Physiol. 575(3): 901-911.

doi:10.1113/jphysiol.2006.112094 
Gibala, M. J. , and McGee, S. L. 2008. Metabolic adaptations to short-term high-intensity interval training: a little pain for a lot of gain? Exerc Sport Sci Rev. 36(2): 58-63. doi:10.1097/JES.0b013e318168ec1f

Gibala, M. J. , Little, J. P., MacDonald, M. J., and Hawley, J. A. 2012. Physiological adaptations to lowvolume, high-intensity interval training in health and disease. J. Physiol. 590(5): 1077-1084. doi:10.1113/jphysiol.2011.224725

Gillen, J. B., and Gibala, M. J. 2014. Is high-intensity interval training a time-efficient exercise strategy to improve health and fitness? Appl Physiol Nutr Metab. 39(3): 409-412. doi:10.1139/apnm2013-0187

Gillen, J.B., Percival, M.E., Ludzki, A., Tarnopolsky, M.A., and Gibala, M.J. 2013. Interval training in the fed or fasted state improves body composition and muscle oxidative capacity in overweight women. Obesity. 21(11): 2249-2255. doi:10.1002/oby.20379

Gist, N.H., Fedewa, M.V., Dishman, R.K., and Cureton, K.J. 2013. Sprint Interval Training Effects on Aerobic Capacity: A Systematic Review and Meta-Analysis. Sports Med. 44(2): 269-279. doi:10.1007/s40279-013-0115-0

Gormley, S. E., Swain, D. P., High, R., Spina, R. J., Dowling, E. A., Kotipalli, U. S., et al. 2008. Effect of intensity of aerobic training on VO2max. Med Sci Sports Exerc. 40(7): 1336-1343. doi:10.1249/MSS.0b013e31816c4839

Hazell, T.J., Hamilton, C.D., Olver, T.D., and Lemon, P.W.R. 2014. Running sprint interval training induces fat loss in women. Appl Physiol Nutr Metab. 39: 944-950. doi:10.1139/apnm-20130503

Hedges, L.V. 1981. Distribution Theory for Glass's Estimator of Effect Size and Related Estimators. J Educ Stat. 6(2): 107-128. doi:10.2307/1164588 
Heydari, M., Freund, J., and Boutcher, S. H. 2012. The Effect of High-Intensity Intermittent Exercise on Body Composition of Overweight Young Males. J Obes. 2012: 1-8. doi:10.1155/2012/480467

Irving, B.A., Davis, C.K., Brock, D.W., Weltman, J.Y., Swift, D., Barrett, E.J., et al. 2008. Effect of exercise training intensity on abdominal visceral fat and body composition. Med Sci Sports Exerc. 40(11): 1863-1872. doi:10.1249/MSS.0b013e3181801d40

Jung, M.E., Bourne, J.E., Beauchamp, M.R., Robinson, E., and Little, J.P. 2015. High-intensity interval training as an efficacious alternative to moderate-intensity continuous training for adults with prediabetes. J Diabetes Res. 2015: 191595. doi:10.1155/2015/191595

Keating, S. E., Machan, E. A., O'Connor, H. T., Gerofi, J. A., Sainsbury, A., Caterson, I. D., et al. 2014. Continuous exercise but not high intensity interval training improves fat distribution in overweight adults. J Obes. 2014: 834865. doi:10.1155/2014/834865

Kelly, B., King, J. A., Goerlach, J., and Nimmo, M. A. 2013. The impact of high-intensity intermittent exercise on resting metabolic rate in healthy males. Eur J Appl Physiol. 113(12): 3039-3047. doi:10.1007/s00421-013-2741-5

Kessler, H. S., Sisson, S. B., and Short, K. R. 2012. The potential for high-intensity interval training to reduce cardiometabolic disease risk. Sports Med. 42(6): 489-509. doi:10.2165/11630910000000000-00000

LaForgia, J., Withers, R. T., and Gore, C. J. 2006. Effects of exercise intensity and duration on the excess post-exercise oxygen consumption. J Sports Sci. 24(12): 1247-1264. doi:10.1080/02640410600552064

Lipsey, M.W., and Wilson, D.B. 2001. Practical meta-analysis. Applied Social Research Methods Series. Sage Publications, Thousand Oaks, CA. 
Macpherson, R. E., Hazell, T. J., Olver, T. D., Paterson, D. H., and Lemon, P. W. 2011. Run sprint interval training improves aerobic performance but not maximal cardiac output. Med Sci Sports Exerc. 43(1): 115-122. doi:10.1249/MSS.0b013e3181e5eacd

Mora-Rodriguez, R., and Coyle, E. F. 2000. Effects of plasma epinephrine on fat metabolism during exercise: interactions with exercise intensity. Am J Physiol Endocrinol Metab. 278(4): E669-676.

Nevill, M. E., Holmyard, D. J., Hall, G. M., Allsop, P., van Oosterhout, A., Burrin, J. M., et al. 1996. Growth hormone responses to treadmill sprinting in sprint- and endurance-trained athletes. Eur J Appl Physiol Occup Physiol. 72(5-6): 460-467. doi:10.1007/BF00242276

Ross, R., Hudson, R., Stotz, P.J., and Lam, M. 2015. Effects of Exercise Amount and Intensity on Abdominal Obesity and Glucose Tolerance in Obese Adults. Ann Intern Med. 162(5): 325-334. doi:10.7326/M14-1189

Rowan, A.E., Kueffner, T.E., and Stavrianeas, S. 2012. Short Duration High-Intensity Interval Training Improves Aerobic Conditioning of Female College Soccer Players. Int J Exerc Sci. 5(3): 232238.

Sandvei, M., Jeppesen, P.B., Støen, L., Litleskare, S., Johansen, E., Stensrud, T., et al. 2012. Sprint interval running increases insulin sensitivity in young healthy subjects. Arch Physiol Biochem. 118(3): 139-147. doi:10.3109/13813455.2012.677454

Sevits, K. J., Melanson, E. L., Swibas, T., Binns, S. E., Klochak, A. L., Lonac, M. C., et al. 2013. Total daily energy expenditure is increased following a single bout of sprint interval training. Physiol Rep. 1(5): e00131. doi:10.1002/phy2.131

Sim, A. Y., Wallman, K. E., Fairchild, T. J., and Guelfi, K. J. 2015. Effects of High-Intensity Intermittent Exercise Training on Appetite Regulation. Med Sci Sports Exerc. 47(11): 24412449. doi:10.1249/mss.0000000000000687 
Sloth, M., Sloth, D., Overgaard, K., and Dalgas, U. 2013. Effects of sprint interval training on VO2max and aerobic exercise performance: A systematic review and meta-analysis. Scand J Med Sci Sports. 23(6): 341-352. doi:10.1111/sms.12092

Subar, A. F., Kirkpatrick, S. I., Mittl, B., Zimmerman, T. P., Thompson, F. E., Bingley, C., et al. 2012. The Automated Self-Administered 24-hour dietary recall (ASA24): a resource for researchers, clinicians, and educators from the National Cancer Institute. J Acad Nutr Diet. 112(8): 11341137. doi:10.1016/j.jand.2012.04.016

Swift, D. L., Johannsen, N. M., Lavie, C. J., Earnest, C. P., and Church, T. S. 2014. The role of exercise and physical activity in weight loss and maintenance. Prog Cardiovasc Dis. 56(4): 441-447. doi:10.1016/j.pcad.2013.09.012

Trapp, E. G., Chisholm, D. J., Freund, J., and Boutcher, S. H. 2008. The effects of high-intensity intermittent exercise training on fat loss and fasting insulin levels of young women. Int J Obes. 32(4): 684-691. doi:10.1038/sj.ijo.0803781

Trilk, J. L., Singhal, A., Bigelman, K. A., and Cureton, K. J. 2011. Effect of sprint interval training on circulatory function during exercise in sedentary, overweight/obese women. Eur J Appl Physiol. 111(8): 1591-1597. doi:10.1007/s00421-010-1777-z

Weston, M., Taylor, K. L., Batterham, A. M., and Hopkins, W. G. 2014. Effects of low-volume highintensity interval training (HIT) on fitness in adults: a meta-analysis of controlled and noncontrolled trials. Sports Med. 44(7): 1005-1017. doi:10.1007/s40279-014-0180-z

Whyte, L. J., Gill, J. M., and Cathcart, A. J. 2010. Effect of 2 weeks of sprint interval training on healthrelated outcomes in sedentary overweight/obese men. Metabolism. 59(10): 1421-1428. doi:10.1016/j.metabol.2010.01.002

Williams, C. B., Zelt, J. G., Castellani, L. N., Little, J. P., Jung, M. E., Wright, D. C., et al. 2013. Changes in mechanisms proposed to mediate fat loss following an acute bout of high-intensity 
interval and endurance exercise. Appl Physiol Nutr Metab. 38(12): 1236-1244.

doi:10.1139/apnm-2013-0101

Zouhal, H., Jacob, C., Delamarche, P., and Gratas-Delamarche, A. 2008. Catecholamines and the effects of exercise, training and gender. Sports Med. 38(5): 401-423 423p. doi:10.2165/00007256200838050-00004 
Table 1. Changes in body composition and aerobic capacity

\begin{tabular}{|c|c|c|c|c|c|c|}
\hline Variable & & $\begin{array}{l}\text { MICT } \\
(n=29)\end{array}$ & $\begin{array}{l}\text { Pairwise } \\
\text { P-value }\end{array}$ & $\begin{array}{c}\text { SIT } \\
(n=23) \\
\end{array}$ & $\begin{array}{l}\text { Pairwise } \\
\text { P-value }\end{array}$ & $\begin{array}{c}\text { GROUP*TIME } \\
\text { P-value }\end{array}$ \\
\hline \multicolumn{7}{|c|}{ Body Mass (kg) } \\
\hline & Baseline & $83.1 \pm 14.2$ & \multirow{3}{*}{.814} & $81.9 \pm 12.2$ & \multirow{4}{*}{.060} & \multirow{3}{*}{.118} \\
\hline & Post & $83.2 \pm 14.6$ & & $81.0 \pm 11.5$ & & \\
\hline & Change & $0.1 \pm 1.8$ & & $-0.9 \pm 2.5$ & & \\
\hline \multicolumn{6}{|c|}{ Fat Mass (kg) } & \\
\hline & Baseline & $36.1 \pm 8.6$ & \multirow{3}{*}{.757} & $33.7 \pm 7.9$ & \multirow{3}{*}{$<.001$} & \multirow{3}{*}{.007} \\
\hline & Post & $35.9 \pm 8.5$ & & $32.5 \pm 7.1$ & & \\
\hline & Change & $0.2 \pm 1.4$ & & $-1.2 \pm 1.9$ & & \\
\hline \multicolumn{7}{|c|}{ Fat Mass $(\%)$} \\
\hline & Baseline & $44.5 \pm 4.8$ & \multirow{3}{*}{.315} & $42.2 \pm 4.8$ & \multirow{3}{*}{$<.001$} & \multirow{3}{*}{.013} \\
\hline & Post & $44.2 \pm 4.4$ & & $41.2 \pm 4.8$ & & \\
\hline & Change & $-0.3 \pm 1.1$ & & $-1.0 \pm 1.4$ & & \\
\hline \multicolumn{7}{|c|}{ Android Fat Mass (kg) } \\
\hline & Baseline & $3.1 \pm 1.0$ & \multirow{3}{*}{.724} & $2.9 \pm 0.9$ & \multirow{3}{*}{$<.001$} & \multirow{3}{*}{.002} \\
\hline & Post & $3.1 \pm 1.0$ & & $2.7 \pm 0.8$ & & \\
\hline & Change & $0.0 \pm 0.2$ & & $-0.2 \pm 0.3$ & & \\
\hline \multicolumn{7}{|c|}{ Whole Body FFM (kg) } \\
\hline & Baseline & $44.5 \pm 6.9$ & \multirow{3}{*}{.149} & $45.6 \pm 5.9$ & \multirow{3}{*}{.208} & \multirow{3}{*}{.983} \\
\hline & Post & $44.8 \pm 7.0$ & & $46.0 \pm 6.3$ & & \\
\hline & Change & $0.4 \pm 1.1$ & & $0.4 \pm 1.5$ & & \\
\hline \multicolumn{7}{|c|}{ Leg FFM (kg) } \\
\hline & Baseline & $24.6 \pm 4.5$ & \multirow{3}{*}{.001} & $25.6 \pm 4.0$ & \multirow{3}{*}{$<.001$} & \multirow{3}{*}{.557} \\
\hline & Post & $25.1 \pm 4.5$ & & $26.1 \pm 4.1$ & & \\
\hline & Change & $0.5 \pm 0.7$ & & $0.6 \pm 0.8$ & & \\
\hline \multicolumn{7}{|c|}{$\mathrm{VO}_{2 \text { peak }}(\mathrm{L} / \mathrm{min})$} \\
\hline & Baseline & $2.2 \pm 0.3$ & \multirow{3}{*}{$<.001$} & $2.3 \pm 0.4$ & \multirow{3}{*}{$<.001$} & \\
\hline & Post & $2.4 \pm 0.3$ & & $2.7 \pm 0.3$ & & .001 \\
\hline & Change & $0.2 \pm 0.2$ & & $0.3 \pm 0.2$ & & \\
\hline $\mathrm{VO}_{2 \text { peak }}($ & & & & & & \\
\hline & Baseline & $26.9 \pm 4.5$ & & $29.1 \pm 4.8$ & & \\
\hline & Post & $28.8 \pm 4.3$ & $<.001$ & $33.2 \pm 4.4$ & $<.001$ & $<.001$ \\
\hline & Change & $1.9 \pm 2.1$ & & $4.1 \pm 3.0$ & & \\
\hline $\mathrm{VO}_{2 \text { peak }}($ & 1/min) & & & & & \\
\hline & Baseline & $50.1 \pm 7.0$ & & $51.6 \pm 7.9$ & & \\
\hline & Post & $53.6 \pm 7.2$ & $<.001$ & $58.4 \pm 6.4$ & $<.001$ & .002 \\
\hline & Change & $3.5 \pm 3.8$ & & $6.8 \pm 4.8$ & & \\
\hline
\end{tabular}

Values are mean \pm SD. FFM $=$ Fat-free mineral-free soft tissue mass. All comparisons controlled for baseline values using Analysis of Covariance with Bonferroni adjustment for multiple comparisons. 


\section{Figure Captions:}

Figure 1. Magnitude of training induced changes in (A) absolute adiposity (kg) and (B) aerobic capacity

$\left(\mathrm{mL} \cdot \mathrm{kg}^{-1} \cdot \mathrm{min}^{-1}\right)$. Values are mean $\pm \mathrm{SEM} . *$ Significant within group difference $(\mathrm{P}<.001) . \dagger$ Significant GROUP*TIME interaction $(\mathrm{P}<.01)$. 
A

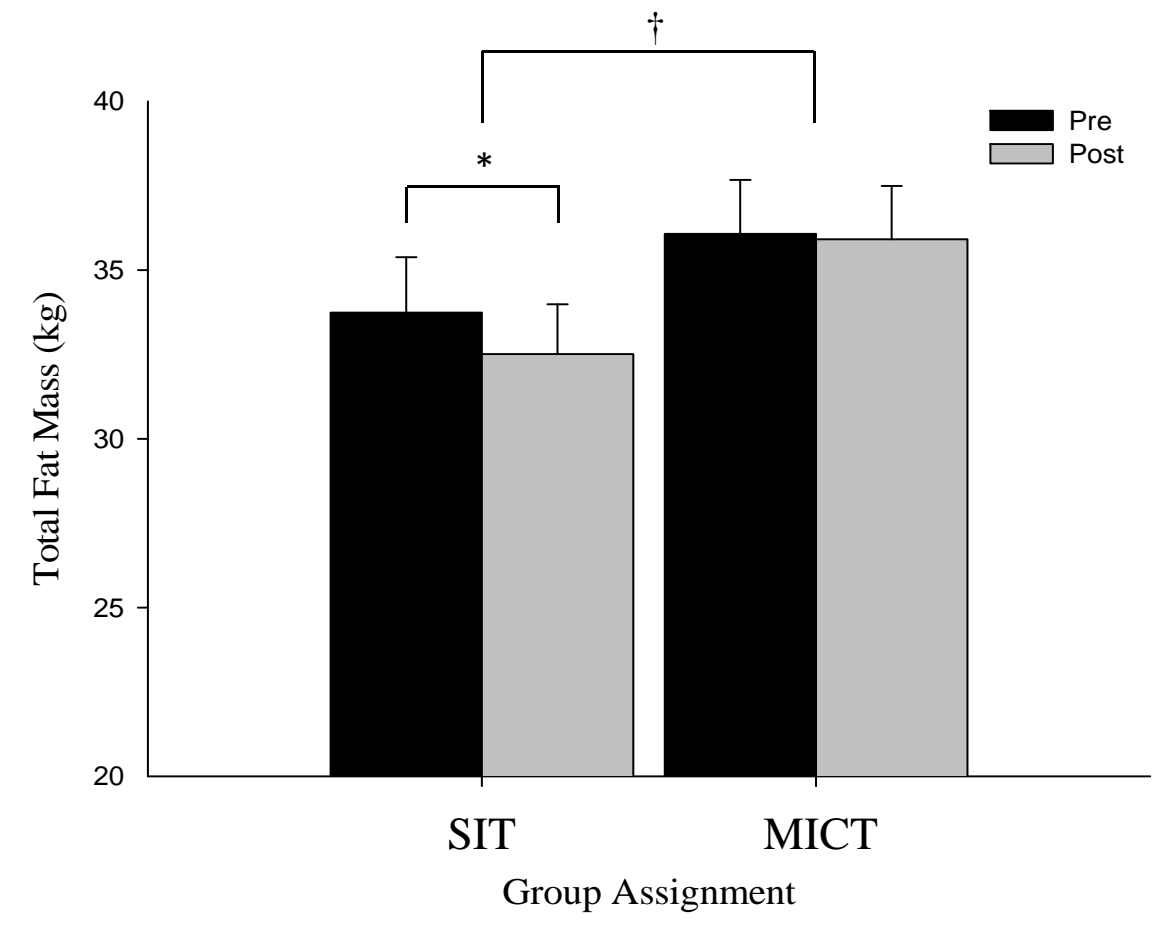

B

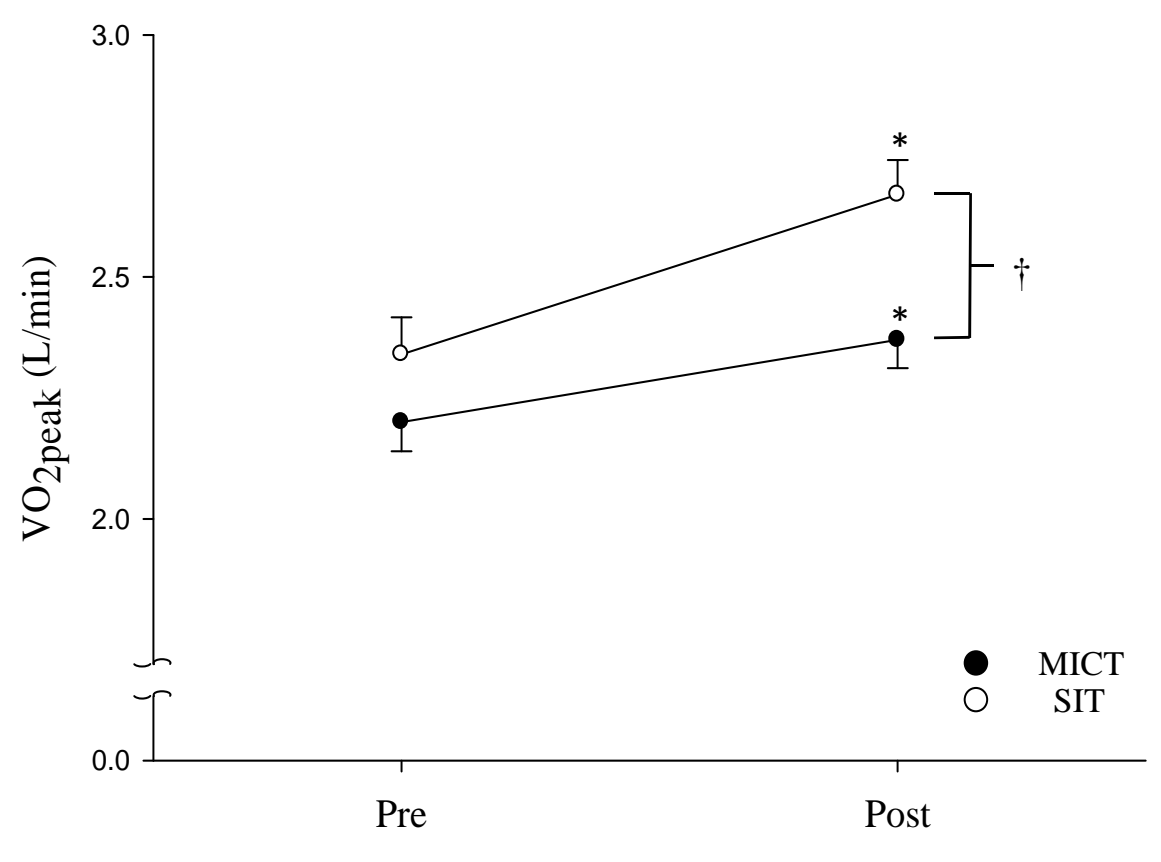

